\title{
Datos de los autores y coordinadores
}

Miquel Bota es doctor en culturas ibéricas y latinoamericanas por Stanford University. Es profesor de Estudios Hispánicos en California State University, Sacramento. Es autor del libro The Contestation of Patriarchy in Luis Martín-Santos' Work (Palgrave, 2020). Su área de investigación y publicación se centra en la revisión y la reinterpretación de la producción cultural ibérica contemporánea, con especial énfasis en los estudios de patriarcado, masculinidades e imperialismo. Como especialista en literatura y cultura contemporáneas sus artículos más recientes incluyen: "The Case of the Catalans: The Forge of an (Anti)Empire" en On the Uses and Abuses of Early Modern Spanish Culture (University of Amsterdam Press 2021); "Ageing and the Rejection of Inheritance in Josep Maria Benet $\mathrm{i}$ Jornet's Testament and Ventura Pons' Amic/Amat" en Ageing Men and Masculinities in Contemporary Spanish Literature, Bulletin of Spanish Studies (Routledge 2021); "Los puentes a la isla de Bergai: El cuarto de atrás o la temporalidad y la estructura del encontrarse," en Del salvaje siglo XIX al inestable siglo XX en las letras peninsulares: una mirada retrospectiva a través de hispanistas (Vernon 2021)"; "E1 23-F de Operación Palace. El (in)movilismo de una 'alegría' televisada," en 2021: La odisea del 23-F (Vervuert 2021); "Cántico: más allá del aire," en Dicenda (2021); "Por la patria y Arráncame la vida: la amistad entre mujeres como elemento (des)estabilizador del status quo" en INTI. Revista de literatura hispánica. Vol. 1. № 93-94 (2021); y "Afectos y política en Mi colegio: una lucha agendada desde el margen," en Artes del ensayo (Spring 2020). Actualmente está trabajando en su segundo libro: The Spanish Pacific: Contemporary Imperial Dilusions of Grandeur y en la edición de Dislocated Empires. Cross-Cultural Perspectives on Spain and the United States.

Juan Carlos Cruz Suárez es doctor en Filología Hispánica por la Universidad de Salamanca y profesor titular en la Universidad de Estocolmo. Sus áreas de investigación principal son las literaturas, el pensamiento y la cultura de la España de los siglos de oro y la literatura memorialista contemporánea de habla hispana. Es autor del libro Ojos con mucha noche. Poesía, pensamiento e ingenio en el Barroco español (Peter Lang, 2014) y ha sido co-editor de tres volúmenes dedicados a los estudios de la literatura y la memoria cultural en el ámbito hispánico (en Peter Lang, 
2012, 2013 y 2014) y de un volumen dedicado a explorar distintas formas de las literaturas hispánicas posnacionales en la era de la globalización (Iberoamericana-Vervuert 2021).

José María Izquierdo es doctor en filosofía por la Universidad de Valencia, España. Actualmente es bibliotecario de investigación y responsable del área de lenguas románicas de la Biblioteca de Humanidades y Ciencias sociales de la Universidad de Oslo. Izquierdo ha sido profesor de literatura en lengua española (1988-2003) en el Departamento de Lenguas Clásicas y Románicas, actual Departamento de literatura, estudios de zona y lenguas europeas de la misma universidad y ha impartido múltiples cursos de formación del profesorado de ELE en Noruega. Su área de investigación se centra en la literatura española. Izquierdo fundó y presidió la Asociación Noruega de Profesores de Español (ANPE-Norge) y fue cofundador y presidió la Federación Internacional de Asociaciones de Profesores de Español (FIAPE). Actualmente es miembro de la dirección de la Asociación de Estudios de Manuel Vázquez Montalbán y uno de los coordinadores de la Red Internacional de Investigación y Aprendizaje: “Memoria y narración”. Es el autor y administrador de los bancos de datos de la Universidad de Oslo: Letras: Traducciones noruegas de literatura en español, Bibliografía sobre la obra de Manuel Vázquez Montalbán y Fremmedspråk og Fremmedspråkundervisning i Norge. Más información: http://www.enmitg.com/wordpress/?page_id=4 y en https://uio.academia.edu/JoseMarialzquierdo.

Claudia Jünke es profesora de literatura y cultura francesa y española en la Universidad de Innsbruck, Austria. Sus investigaciones se centran en las literaturas y culturas modernas y contemporáneas en Francia, España y Latinoamérica, con un enfoque particular en la memora cultural de pasados violentos, el nexo entre memoria y traducción, identidades culturales, narratología e intermedialidad. Sus publicaciones incluyen un libro monográfico sobre la memoria de la guerra civil española en la literatura y el cine (2012); un libro coeditado sobre la Movida madrileña como fenómeno transmedial (2014); y el dossier Juana Inés de la Cruz: identidad criolla y procesos de transculturación (2019).

Adriana Minardi es doctora por la Universidad de Buenos de Aires e Investigadora adjunta del CONICET (Consejo Nacional de Investigaciones Científicas y Técnicas). Ha publicado trabajos sobre la producción ensayística y narrativa de Juan Benet en diversas revistas académicas con 
referato y participó como editora y autora del libro La escritura íntima de Manuel Azaña. Ha publicado Los mensajes de fin de año de Francisco Franco. Un análisis ideológico-discursivo, por la editorial Biblos, 2010, Memoria, historia, discurso. Variaciones sobre algunos ensayos benetianos, Pliegos, 2013 y Formas del ensayo en Juan Benet, Eudeba, 2020. Ha sido investigadora externa del proyecto "La memoria novelada" de la Universidad de Aarhus, Dinamarca y directora del proyecto PICT "Memoria, Escritura, Retórica política. Manuel Azaña y Juan Benet. Derroteros críticos de la paradoja hispánica". Sus áreas de trabajo son la literatura española contemporánea y el análisis del discurso, con especial foco en temas como la relación retórica/política, la memoria discursiva y la construcción del ethos. Ha sido becaria de la Fundación Carolina y ha dictado conferencias en la Universidad de Aarhus, en la Universidad de Kassel- de la que también ha sido becaria posdoctoral-, Universidad de Valladolid y en la Universidad Federal de Río Grande do Sul en el equipo del SEAD (Seminario de Estudios en Análisis del Discurso). Integra actualmente el comité de redacción de la revista Filología (CIRC-LATINDEX) y es asistente editorial de la revista Rétor (CIRC) así como vocal de la Asociación Argentina de Retórica. Actualmente es directora de un proyecto PIP sobre la producción literaria española de los llamados "felices años veinte". Es directora de una becaria doctoral en la Universidad de Buenos Aires y de diversos adscriptos en la cátedra de Literatura española III de la misma universidad. Ha incursionado en líneas originales respecto de la construcción política de la memoria, en especial en lo que llama narrativas "de crisis" en lo que tienen de componente programático y de función argumentativa a partir de tres ejes: a) la construcción biopolítica de las representaciones con su puesta textual basada en la biorretórica b) la noción de verdad en relación al discurso de la historia y c) la función de la imaginación en términos de memoria que opera como repliegue de una comunidad imaginada. Esta tríada funciona, además, como paradigma metodológico que nos permitirá estudiar la noción de crisis desde la Retórica (Barei 2012, Pain 2002, Villacañas 2013) y la Sociocrítica (Arfuch 2003, Koselleck 2007) para asumir este concepto en términos de un "significante flotante" que designará diferentes variaciones según los paradigmas de autoridad a los que se enfrente la novela como género dominante. La crisis supone la ruptura de los marcos institucionales, morales, sociales y cognitivos. De alguna manera, la crisis es una función que temporaliza y construye subjetividades. La semántica de la crisis proporciona elementos necesarios para pensar las disrupciones, las distopías y los engranajes en su condición de instrumental teórico-metodológico. La noción de verdad 
asume el desplazamiento semántico a la ficción como una nueva forma de recuperación de la memoria colectiva (Halbwachs 1968) que tiene por objeto, buscar la integración del saber, sentir, actuar, colectivos (Winter 2006). De ahí que la representación de la crisis sea, ante todo, ideológica. ¿En qué consiste la relación entre la experiencia de la crisis y la práctica de la crítica memorialista? Es decir, cómo la literatura interviene en la construcción de los lugares de memoria (Nora, 1989). Resulta clave indagar los procesos argumentativos de la ficción según los ejes descriptos de la corporalidad, la metaficción histórica y la memoria.

Jordi Osúa Quintana es doctor por la Universidad de Barcelona con una tesis dedicada a "El deporte en la vida y en la obra de Manuel Vázquez Montalbán (1939-2003)". Es autor de "Manuel Vázquez Montalbán. Barça, cultura i esport", "Vázquez Montalbán: fútbol y política" (Premio Becerro de Bengoa), "Esport contemporani i socialitat postmoderna: entre la identitat i la globalització" (Premio Serra i Moret) y de la guía didáctica y de lectura para ESO y Bachillerato "El delantero centro fue asesinado al atardecer". Ha publicado diversos artículos sobre Manuel Vázquez Montalbán y el deporte en revistas especializadas y en diarios.

Anne-Sophie Owczarczak es doctora en Estudios Hispánicos, profesora de español asociada en la Universidad de Valenciennes (Francia) y en la enseñanza secundaria. Miembro del laboratorio Textes et Cultures (EA4028), equipo interno CoTraLiS en Arras. Su tesis, defendida en junio de 2019, trata del impacto de las palabras escritas por Manuel Vázquez Montalbán en las revistas Por Favor y Triunfo en 1975, bajo un enfoque lexicológico y discursivo. Temas de investigación: Manuel Vázquez Montalbán, prensa del franquismo, análisis del discurso, memoria histórica, historia de la dictadura y de la transición.

Santiago Sevilla Vallejo es profesor Ayudante Doctor en la Universidad de Salamanca. Ha sido secretario de la Federación de Asociaciones de Profesores de Español y es director de la revista Cálamo FASPE. Dirige el Congreso Internacional Las Desconocidas. Estudios sobre la construcción de la identidad femenina en la literatura, que forma parte del grupo de investigación Escritoras y personajes femeninos en la literatura (Universidad de Salamanca). Ha sido finalista del III Premio Educa Abanca. Mejor Docente de España, en la categoría Universidad; y del premio de investigación de la Primera edición del Congreso Internacional de Escritores y Artistas. 